\title{
L'Église dansante. Initiation, rituel et liturgie en France au bas Moyen Âge
}

Thèse de doctorat en histoire sous la direction de Neithard Bulst, université de Bielefeld, Allemagne. Soutenue le 15 avril 2014

Eine tanzende Kirche. Initiation, Ritual und Liturgie im spätmittelalterlichen

Frankreich

\section{Philip Knäble}

\section{OpenEdition \\ Journals}

Electronic version

URL: https://journals.openedition.org/cem/14328

DOI: $10.4000 /$ cem. 14328

ISSN: 1954-3093

Publisher

Centre d'études médiévales Saint-Germain d'Auxerre

Electronic reference

Philip Knäble, "L'Église dansante. Initiation, rituel et liturgie en France au bas Moyen Âge", Bulletin du centre d'études médiévales d'Auxerre | BUCEMA [Online], 20.1 | 2016, Online since 14 June 2016, connection on 22 September 2022. URL: http://journals.openedition.org/cem/14328 ; DOI: https:// doi.org/10.4000/cem. 14328

This text was automatically generated on 22 September 2022

\section{(c) (i) (8)}

Creative Commons - Attribution-NonCommercial-ShareAlike 4.0 International - CC BY-NC-SA 4.0 https://creativecommons.org/licenses/by-nc-sa/4.0/ 


\section{L'Église dansante. Initiation, rituel et liturgie en France au bas Moyen Âge}

Thèse de doctorat en histoire sous la direction de Neithard Bulst, université de Bielefeld, Allemagne. Soutenue le 15 avril 2014

Eine tanzende Kirche. Initiation, Ritual und Liturgie im spätmittelalterlichen

Frankreich

Philip Knäble

1 Les relations entre l'Église et la danse à la fin du Moyen Âge ont été étudiées par plusieurs disciplines, entre autres l'ethnologie, la théologie ou la musicologie, mais très peu par l'histoire médiévale. À contre-courant de l'opinion fréquente d'une Église hostile à la danse, la thèse de doctorat présentée ici pose la question de savoir comment et sous quelles conditions la danse faisait partie

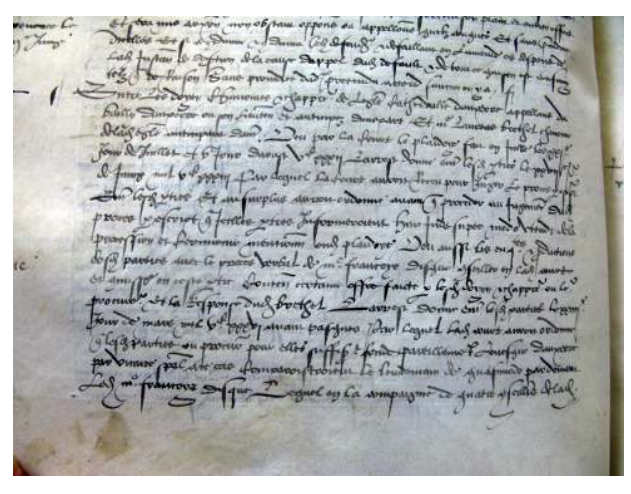
de la religiosité $\mathrm{du}$ bas Moyen Âge.

L'hypothèse centrale est que les pratiques de danse n'étaient ni des reliquats païens ni uniquement des manifestations d'une culture populaire profane, mais, qu'au contraire, elles existaient au sein même de l'Église. Le contexte clérical des danses est signifié premièrement par une date du calendrier religieux, deuxièmement par un espace sacré comme une église, un cimetière ou un espace temporairement sacralisé par une procession et, troisièmement, par la participation des clercs aux danses.

2 L'étude se concentre sur deux communautés de chanoines en France, les chapitres cathédraux d'Auxerre et de Sens. Les deux chapitres ont été choisis parce qu'ils sont situés dans la même province ecclésiastique, leurs églises sont dédiées au même patron, 
saint Étienne, et ils partageaient une similarité architecturale : un grand dédale au centre de la nef, comme il en existe aujourd'hui encore à Chartres. De plus, les danses, la Pelotte ${ }^{1}$ à Auxerre et la Cazzole ${ }^{2}$ à Sens, avaient lieu le jour de Pâques.

3 Suivant l'exemple des ritual studies, des performance studies et le concept d'une "société de présence" (Anwesenheitsgesellschaft) ${ }^{3}$, la thèse analyse comment la pratique corporelle de la danse permet de symboliser l'harmonie céleste et l'initiation des nouveaux chanoines dans les chapitres. L'étude met en lumière les légitimations ambivalentes des danses, la négociation du pouvoir entre les chapitres cathédraux et leur évêque par la danse et l'évolution des danses religieuses au début de l'époque moderne.

\section{L'Église dansante?}

4 Tout d'abord se pose la question de savoir d'où vient l'image d'une Église médiévale complètement opposée à la danse. Certes, des études issues de plusieurs disciplines ont traité la relation entre l'Église et la danse, mais un examen détaillé des sources médiévales et modernes reste l'exception. La thèse commence donc par l'histoire de la perception de la danse religieuse jusqu'à nos jours afin de reconstituer les différents interprétations et jugements sur ce phénomène.

5 Une analyse nuancée des traités de danses et des décisions de conciles indique que la danse, dans le contexte clérical du Moyen Âge tardif, n'était pas un corps étranger, mais qu'au contraire elle faisait, sous de multiples formes, partie intégrante de la religiosité médiévale. Le jugement sur les danses porté par les clercs est donc exceptionnellement ambivalent. Outre les ecclésiastiques qui critiquent les danses en bloc, il y a aussi des auteurs qui distinguent les danses selon leurs motifs, occasions ou formes de mouvement, et même ceux qui considèrent la danse comme un moyen d'atteindre l'harmonie céleste plaisant à Dieu. Une condamnation totale des danses par l'Église n'existe ni avant la Réforme ni même chez les clercs catholiques, luthériens et calvinistes $\mathrm{du} \mathrm{xvI}^{\mathrm{e}}$ siècle ${ }^{4}$. Ce n'est qu'au cours du siècle suivant que les danses religieuses, considérées comme scandaleuses et arriérées, tombent dans l'oubli, tant et si bien que se cristallise le cliché d'une religiosité médiévale sévère et excluant toute pratique corporelle ou ludique.

6 Pourtant, l'idée d'une Église médiévale opposée à la danse est remise en question par le fait que souvent les promoteurs supposés des interdictions, les cardinaux, les évêques, les abbesses et abbés eux-mêmes dansaient et organisaient les spectacles de danse. La danse était une activité tellement liée aux fêtes aristocratiques et bourgeoises et constituait une partie si importante de la communication symbolique, et donc politique, que le clergé ne pouvait y échapper. Les spectacles en rapport avec les assemblées cléricales, par exemple le Concile de Trente (1545-1563), étaient régulièrement fréquentés par des clercs dansants. À côté des spectacles laïques, il y avait aussi de nombreuses fêtes religieuses, comme des processions, des rites de passage ou des mystères, où des clercs et des fidèles dansaient. Ce sont surtout les chapitres cathédraux et collégiaux en France qui intègrent les danses au déroulement des solennités comme Noël, Pâques ou la Pentecôte. En fonction du calendrier liturgique local, le choix des danseurs et danseuses, les mouvements autorisés et la légitimation théologique varient fortement. C'est pourquoi les danses à Auxerre et à Sens ne sont pas - comme l'estiment les auteurs du XVIII ${ }^{\mathrm{e}}$ siècle, notamment le chanoine 
d'Auxerre Jean Lebeuf - une "grande bizarrerie $»^{5}:$ au contraire, les danses représentent une forme de communication symbolique par laquelle les chapitres et leurs évêques négocient la structure du pouvoir local.

\section{La Pelotte d'Auxerre et la Cazzole de Sens}

7 Les deux cas étudiés, les chapitres cathédraux d'Auxerre et de Sens, étaient de grandes communautés prospères. Les chapitres et les évêques se partageaient le gouvernement temporel et spirituel du quartier cathédral ${ }^{6}$. Des coutumes écrites fixaient les droits, obligations et privilèges des deux parties, et leur interprétation déclenchait souvent des conflits. L'accès à la cathédrale, la participation aux messes, les vêtements, les gestes et les mouvements dans le quartier cathédral étaient tous des aspects sévèrement réglementés et dont le non-respect était bruyamment critiqué. Les danses dans la nef de la cathédrale à Auxerre et la danse sur la place du Cloître à Sens devaient inévitablement influer sur la fragile relation entre le chapitre et l'évêque, d'autant plus qu'elles avaient lieu durant la fête la plus importante du christianisme.

Il est impossible de dater les débuts des danses à Auxerre et à Sens par suite du manque de sources. Toutefois, les traités des liturgistes comme Jean Beleth ( $\dagger 1165)$, Sicard de Crémone $(\dagger 1215)$ et Guillaume d'Auxerre $(\dagger 1231)$ indiquent que ces danses font probablement partie des pratiques de danse très élaborées qui se développent dans les églises cathédrales en France aux XII ${ }^{\mathrm{e}}$ et XIII ${ }^{\mathrm{e}}$ siècles ${ }^{7}$. Les liturgistes décrivent, en effet, plusieurs danses, jeux des ballons ou mélanges de jeux et danses, que les chanoines des cathédrales françaises effectuent le jour de Pâques. La réception du concept platonicien de l'harmonie des sphères dans les écoles cathédrales et son adaptation à la cosmologie chrétienne permettent aux théologiens une interprétation positive de la danse dans un contexte religieux. L'idée platonicienne de la ronde des astres, réinterprétée comme la ronde des anges ou des saints, fait de la danse une légitime activité de dévotion. En même temps, les clercs des écoles cathédrales redécouvrent les exemples bibliques de la danse de David devant l'Arche sacrée ou de Myriam après le passage de la mer Rouge, et s'en inspirent pour leurs danses, spirituelles, mais aussi, de plus en plus, corporelles. En France, ces réflexions des clercs sur la danse divine harmonieuse donnent lieu à plusieurs formes de danses durant les solennités chrétiennes, dont la Pelotte d'Auxerre et la Cazzole de Sens sont deux variantes.

9 À Sens, la procession aux fonts baptismaux - qui a lieu dans de nombreuses églises le jour de Pâques - devient sous la conduite des chanoines une procession dansante. Les chanoines, précédés par l'archevêque, avancent en deux rangées jusqu'aux fonts en chantant des hymnes de la Résurrection, probablement suivis par des danseurs laïques. La danse n'avait pas lieu, comme plusieurs études le prétendent, sur le labyrinthe dans la nef de l'église, mais sur la place du Cloître à l'extérieur de la cathédrale ${ }^{8}$. Un manuscrit au début du Xvi ${ }^{\mathrm{e}}$ siècle souligne que la procession dansante s'effectue sans sauts ( [...] non tamen saliendo [...] ${ }^{9} »$ ) comme il serait le cas lors des danses scandaleuses, mais au contraire par des mouvements graves et modestes. Lorsque la participation des laïcs des deux sexes à la danse devient de plus en plus fréquente, les chanoines décident en 1519 de déplacer la procession dans le chœur de la cathédrale et d'y bannir les danses.

10 À Auxerre, la danse fait partie d'un rite de passage élaboré pendant laquel un nouveau chanoine est accueilli au sein de la communauté. La Pelotte est un mélange de danse et 
de jeu de ballon, que le chapitre exécutait sur le labyrinthe dans la nef de la cathédrale. Après avoir reçu du nouveau chanoine un grand ballon, la Pelotte, le doyen prend celleci de sa main gauche et danse au centre du labyrinthe, tandis que les chanoines, se tenant par la main, se meuvent autour du dédale. Puis le doyen lance le ballon aux chanoines qui dansent alors en chaîne à côté de lui en chantant l'antienne Victimae Paschali laudes ${ }^{10}$. Cette chorégraphie fait allusion au mythe grec de Thésée adapté dans un contexte chrétien - la victoire de Thésée sur le minotaure étant interprétée comme la victoire du Christ sur Satan et sa Résurrection le jour de Pâques - et à la création du monde ${ }^{11}$. Ce sont deux sujets qui sont évoqués pendant la liturgie du jour de Pâques, qui est en France jusqu'au $\mathrm{XvI}^{\mathrm{e}}$ siècle aussi le début de la nouvelle année, et donc d'une nouvelle ronde céleste. L'association d'une danse ecclésiastique avec le lancer de ballon dans la cathédrale d'Auxerre le jour de Pâques représentait donc la mise en scène christianisée de l'harmonie des sphères.

11 En présence du gouvernement laïque d'Auxerre, représenté par le gouverneur et le bailli de la cité, mais en l'absence des autres communautés ecclésiastiques et de l'évêque, le chapitre signalait aussi sa position particulière dans le gouvernement de la ville. Comme une analyse des dossiers redécouverts au Parlement de Paris le souligne ${ }^{12}$, ce n'est point la pression d'acteurs externes, mais les dissensions internes dans le chapitre qui provoquent la fin de la Pelotte au milieu du xvI siècle. Quand le procès entre le chapitre et un chanoine qui refuse à danser est porté devant le Parlement de Paris, les théologiens de la Sorbonne et les clercs du Parlement de Paris n'hésitent pas à interdire un rituel qui leur paraît superstitieux, d'autant plus qu'ils craignent la critique des réformateurs.

Par la conclusion que la danse pouvait être, sous certaines conditions, une pratique religieuse reconnue à la fin du Moyen Âge, la thèse permet une réévaluation de la culture festive religieuse de cette époque. Comme le montrent également des travaux récents sur la Fête des Fous, la Manie dansante, la Fête de l'Enfant-évêque ou des jeux monastiques $^{13}$, l'Église médiévale acceptait les nombreuses formes de pratiques corporelles ludiques comme une expression autorisée de la foi en les légitimant par la théologie. Les jeux et les danses ne faisaient certes jamais directement partie de la liturgie, mais ils s'y référaient toujours et se déroulaient juste avant ou après la liturgie proprement dite : on peut - en référence au germaniste Jan-Dirk Müller - désigner ces pratiques comme "paraliturgiques $»^{14}$. La thèse apporte ainsi une contribution aux débats actuels sur le caractère corporel de la religiosité.

Reçu : 28 février 2016 - Accepté : 10 avril 2016

\section{NOTES}

1. Par le mot Pelotte (Pelote, lat. pilota) les sources médiévales désignent ou le ballon ou le rituel entier à Auxerre. Cf. PARIS, Archives nationales, X1a 1541, fol. 435 $\mathrm{r}^{\circ}$; Archives départementales de l'Yonne, G 1798, fol. 285 ; J. LEBEUf, "Explication d'un terme de la basse Latinité ", Mercure de France, 10 (mai 1726), p. 911-925; JEAN LEBEUF, Mémoires concernant l'histoire civile et ecclésiastique 
d'Auxerre et son ancien diocèse, éd. A. CHALlE et M. QUANTIN, 4 vol., Auxerre, 1848-1855, t. 4, p. 322 sq.; C. WRIGHT, The Maze and the Warrior: Symbols in Architecture, Theology and Music, Cambridge, 2001, p. 321.

2. Le nom Cazzole est utilisé pour la danse à Sens pour la première fois par le chanoine JeanBasile-Pascal Fenel au milieu du XVIII ${ }^{\mathrm{e}}$ siècle, J. LEBEUf, Lettres de l'abbé Lebeuf, éd. A. CHEREST et M. QUANTIN, 2 vol., Auxerre, 1866-1867, t. 2, p. 289.

3. R. SCHLÖGL, « Kommunikation und Vergesellschaftung unter Anwesenden. Formen des Sozialen und ihre Transformation in der Frühen Neuzeit », Geschichte und Gesellschaft, 34 (2008), p. 155-224.

4. A. WÉRY, La danse écartelée de la fin du Moyen Âge à l'âge classique, Paris, 1992 ; M. RUEL, Les chrétiens et la danse dans la France moderne : XVI ${ }^{e}-\mathrm{XVIII}{ }^{e}$ siècle, Paris, 2006 ; M.-J. LOUISON-LASSABLIÈRE, Feuillets pour Terpsichore. La danse par les textes $d u \mathrm{XV}^{e}$ au XVII siècle, Paris, 2007.

5. J. LEBEUF, « Explication d'un terme de la basse Latinité », Mercure de France, 10 (mai 1726), p. 918.

6. Sur Auxerre, cf. c. SAPIN, Saint-Étienne d'Auxerre. La seconde vie d'une cathédrale, Paris, 2011 ; C. DEMAY, "L'évêque d'Auxerre et le chapitre cathédral au XVIII ${ }^{\mathrm{e}}$ siècle ", Bulletin de la Société des sciences de l'Yonne, 52 (1898), p. 7-226; sur Sens, cf. D. CAILlEAUX, La cathédrale en chantier. La construction du transept de Saint-Etienne de Sens d'après les comptes de la fabrique 1490-1517, Paris, 1999 ; V. TABBAGH (dir.), Diocèse de Sens, Turnhout, 2010 (Fasti Ecclesiae Gallicanae, 11).

7. Cf. C. MEWs, «Liturgists and Dance in the Twelfth Century: The Witness of John Beleth and Sicard of Cremona ", Church History, 78/3 (September 2009), p. 512-548.

8. Cf. POITIERS, Bibliothèque municipale, ms. 336 , fol. $90 \mathrm{r}^{\circ}$; AUXERRE, Bibliothèque municipale, ms. 207, fol. 24 ; J. Lebeuf, Lettres..., op. cit., t. 2, p. 289 ; D. CAIllEAuX « Diocèse de Sens », in V. TABBAGH (dir.), Diocèse de Sens..., op. cit., p. 36.

9. Cf. POITIERs, Bibliothèque municipale, ms. 336, fol. $90 \mathrm{r}^{\circ}$.

10. J. LEBEUf, « Explication d'un terme... ", op. cit., p. 921 sq.

11. C. WRIGHT, The Maze and the Warrior..., op. cit. ; M. EISENBERG, "Performing the Passion : Music, Ritual, and the Eastertide Labyrinth ", Revista Transcultural de Música/Transcultural Music Review, 13 (2009), sans pagination.

12. PARIS, Archives nationales, $\mathrm{X} 1 \mathrm{a} 1541$, fol. $434 \mathrm{v}^{\circ}-435 \mathrm{r}^{\circ}$.

13. M. HARRIS, Sacred Folly. A New History of the Feast of Fools, Ithaca, 2011 ; G. ROHMANN, Tanzwut. Kosmos, Kirche und Mensch in der Bedeutungsgeschichte eines mittelalterlichen Krankheitskonzeptes, Göttingen, 2013 ; Y. DAHHAOUI, « Voyages d'un prélat festif. Un "évêque des Innocents" dans son évêché ", Revue historique, 308 (2006), p. 677-694 ; T. SKAMBRAKS, Das Kinderbischofsfest im Mittelalter, Tavarnuzze, 2014 ; J. SonNTAG (dir.), Religiosus ludens. Das Spiel als kulturelles Phänomen in mittelalterlichen Klöstern und Orden, Berlin, 2013.

14. J.-D. MÜLLER, «Ritual, pararituelle Handlungen, Geistliches Spiel. Zum Verhältnis von Schrift und Performanz », in w. HORST (dir.), Audiovisualität vor und nach Gutenberg. Zur Kulturgeschichte der medialen Umbrüche, Vienne, 2001, p. 63-71.

\section{INDEX}

Geographical index: France/Auxerre, France/Sens

Mots-clés: danse, liturgie, chapitres cathédraux, Pelotte 
AUTHOR

\section{PHILIP KNÄBLE}

Universität Göttingen. Postdoktorand. Graduiertenkolleg « Expertenkulturen des 12. bis 18.

Jahrhunderts » 